\title{
Relationship between HER2 Proto-oncogene Status and Prognostic Factors of Breast Cancer in the West of Iran
}

\author{
Nasrin Amirifard ${ }^{1}$, Edris Sadeghi ${ }^{1 *}$, Mehrdad Payandeh ${ }^{2}$, Hossain Mohebbi ${ }^{3}$, \\ Masoud Sadeghi ${ }^{3}$, Mansour Choubsaz ${ }^{1}$
}

\begin{abstract}
Background: Breast cancer is a very common health problem in Iranian women. The HER2-neu gene is a transmembrane receptor tyrosine kinase with homology to members of the EGF receptor family. The aim of this study was to investigate the association between HER2-neu oncogene status with prognostic factors of breast cancer in Kermanshah province, Iran. Materials and Methods: Relationship between HER2-neu and prognostic factors of 130 cases of breast cancer were evaluated during two years in Imam Reza hospital in Kermanshah, Iran. Data were analyzed using descriptive statistics and the T-test and Mann-Whitney U non-parametric test

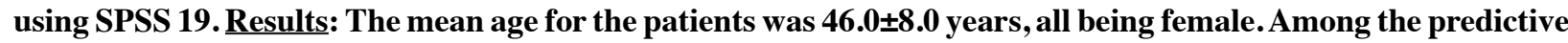
factors for breast cancer were family history, stage of disease, involvement of the lymphovascular system, number of involved lymph nodes in axillaries, grading and hormone receptor status with HER2-neu oncogene had direct correlation and between factors, tumor location, patient age and histological characteristics and HER2-neu oncogene had no significant relationship. We found significant correlation between HER2 with ER and PR and also HER2 with ER, PR negative. Conclusions: HER2-neu is risk factor that can be a good prognostic and also predictive factor. For these reasons, we recommend that it be evaluated for all types of $\mathrm{BC}$.
\end{abstract}

Keywords: Breast cancer - HER2 - prognostic factors - trastuzumab

Asian Pac J Cancer Prev, 17 (1), 295-298

\section{Introduction}

Breast cancer $(\mathrm{BC})$ is the most common health problem in Iranian women that its incidence is increasing rapidly. It is the fifth cause of death and third burden of disease among women malignant disease (Mohammadi et al., 2013). Prognostic factors (well defined and presumptive, anatomic and biochemical, and clinical and molecular) influence the design, conduct, and analysis of clinical trials of early-stage breast cancer (Dorr, 1993). In addition to, prognostic factors such as age, tumor size, and cancer stage and grade, overexpression of the human epidermal growth factor receptor 2 (HER2)-positive and positivity for estrogen receptor (ER) or progesterone receptor (PR) are heavily studied prognostic (Qin et al., 2015). In BC; ER and PR were the first predictive molecular markers (Payandeh et al., 2015). However, 25-50\% of ER and PR positive tumors are resistant to hormone therapy (Klijin, 1991), and tumors with HER-2 overexpression do not always respond to trastuzumab therapy. In addition, about $20 \%$ of patients with BC are negative for all three markers mentioned above, and their tumors are more resistant to traditional therapy schemes (Yan et al., 2015). However, breast cancers have heterogeneous features that are diverse in behavior, outcome and response to therapy. It is difficult to predict outcomes in all breast cancer patients using traditional histopathologic factors and the same biomarkers factors (Dowsett and Dunbier, 2008). In studies suggested a categorization of invasive breast cancers based on genetic profiles into five subtypes with clinical implications: luminal A, luminal B, HER-2/neu overexpressing, normal breast like and triple negative phenotype (Perou et al., 2000; Sotiriou et al., 2003). The HER-2/neu gene encodes a $185-\mathrm{kDa}$ transmembrane receptor tyrosine kinase with homology to members of the EGF receptor family. Unlike the other EGF receptors, HER-2/neu has an intrinsic tyrosine kinase activity that activates receptor-mediated signal transduction in the absence of ligand (Hu et al ., 1992). The immunohistochemical staining for steroid hormone receptors (ER and PR, respectively) and the human epidermal growth factor receptor-2 (HER2) helps to choose appropriate therapies and predict prognosis for breast cancer patients (Doval et al., 2015). A positive HER-2 status in patients with BC correlates with high sensitivity to targeted therapy with trastuzumab. These biomarkers play an important role in clinical practice by individualizing treatment and determining adequate 
Nasrin Amirifard et al

chemotherapeutic schemes for patients (Yan et al., 2015). In some studies, the prognostic value of HER-2/neu was not independent of cell-proliferation rate ( $\mathrm{Ji}$ et al., 1993) or other markers of tumor aggressiveness (Hartmann et al., 1994). The aim of this study was to investigate the association HER-2/neu oncogene in breast cancer predictive factors in Kermanshah province, Iran.

\section{Materials and Methods}

The relationship between HER-2/neu and prognostic factors of 130 cases of breast cancer was evaluated during two years in Imam Reza hospital in Kermanshah, Iran. The data were analyzed using descriptive statistics IBM SPSS v. 19 (T-test, Chi-square and Mann-Whitney test).

Table 1. The Baseline Characteristics for All Patients

\begin{tabular}{|c|c|c|}
\hline Variables & $\mathrm{n}(\%)$ & Mean \pm SD \\
\hline Age (year) & & $46.02 \pm 8.04$ \\
\hline $20-30$ & $6(4)$ & \\
\hline $31-40$ & $32(25)$ & \\
\hline $41-50$ & $56(43)$ & \\
\hline$>51$ & $36(28)$ & \\
\hline \multicolumn{3}{|l|}{ HER2-neu } \\
\hline Positive & $47(36)$ & \\
\hline Negative & $83(64)$ & \\
\hline \multicolumn{3}{|l|}{ Family History } \\
\hline Positive & $48(37)$ & \\
\hline Negative & $82(63)$ & \\
\hline \multicolumn{3}{|l|}{ Stage } \\
\hline 0 & $11(9)$ & \\
\hline I & $16(12)$ & \\
\hline II & $55(42)$ & \\
\hline III & $38(29)$ & \\
\hline IV & $10(8)$ & \\
\hline \multicolumn{3}{|l|}{ Histological Features } \\
\hline Ductal carcinoma & $104(80)$ & \\
\hline Lobular carcinoma & $16(12)$ & \\
\hline Other & $10(8)$ & \\
\hline \multicolumn{3}{|l|}{ Lymph node involvement } \\
\hline Yes & $85(65)$ & \\
\hline No & $45(35)$ & \\
\hline \multicolumn{3}{|l|}{ Number of lymph nodes } \\
\hline 0 & $45(35)$ & \\
\hline $1-3$ & $51(39)$ & \\
\hline $4-9$ & 25(19) & \\
\hline$>9$ & $9(7)$ & \\
\hline \multicolumn{3}{|l|}{ Grade } \\
\hline I & $17(13)$ & \\
\hline II & $100(77)$ & \\
\hline III & $13(10)$ & \\
\hline
\end{tabular}

\section{Results}

The mean age for the patients at diagnosis was $46.02 \pm 8.04$ years, $100 \%$ female. Six patients $(4 \%)$, thirty two $(25 \%)$, fifty sex $(43 \%)$ and thirty sex $(28 \%)$ were located in age group of 20 to 30,31 to 40,41 to 50 and $51+$, respectively. Forty-seven patients $(36 \%)$ had HER2positive and eighty three (64\%) had HER2-negative. The most patients according to age, HER2-neu, family history, stage, histological features, lymph node involvement, number of lymph nodes and grade were 41-50 years, negative, negative, stage II, ductal carcinoma, yes, 1 to 3 lymph nodes and grade III, respectively. The lowest frequency of patients according to age, stage, number of lymph nodes and grade had 20-30 years, stage IV, >9 lymph nodes and grade II, respectively (Table 1).

The most frequency tumor location was belonging to upper outer of breast and the less frequency was belonging to inner and axillary tail of breast (Figure 1).

Among the $\mathrm{BC}$ predictive factors like to family history, stage, lymph node involvement, number of

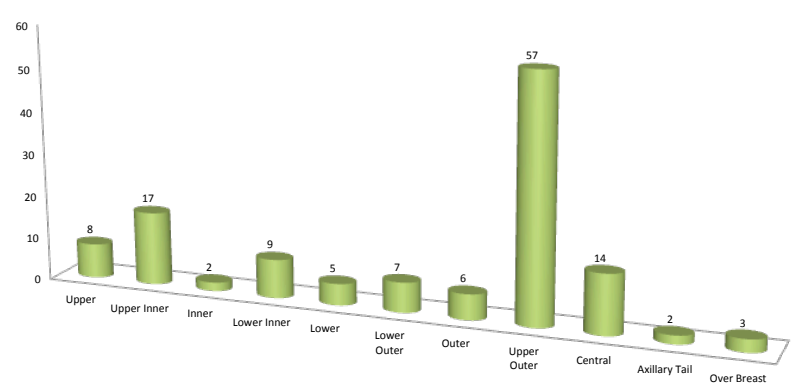

Figure 1. The Number of Patients Based on Tumor Location

Table 3. The Variables in the Patients Based on HER2

\begin{tabular}{lrrc}
\hline Variable & $\begin{array}{c}\mathrm{n}(\%) \\
{[\mathrm{HER} 2+]}\end{array}$ & $\begin{array}{c}\mathrm{n}(\%) \\
{[\text { HER2-] }}\end{array}$ & $\mathrm{P}$ \\
\hline \multicolumn{1}{l}{ Histological Features } & & & 0.908 \\
$\quad$ Ductal carcinoma & $47(36)$ & $57(44)$ & \\
$\quad$ Lobular carcinoma & $0(0)$ & $16(12)$ & \\
$\quad$ Other & $0(0)$ & $10(8)$ & \\
$\begin{array}{l}\text { Marker } \\
E R\end{array}$ & & & \\
$\quad$ Positive & $13(19.6)$ & $53(80.4)$ & 0.001 \\
$\quad$ Negative & $15(22.7)$ & $49(77.3)$ & 0.031 \\
$P R$ & & & \\
$\quad$ Positive & $12(16.3)$ & $64(88.7)$ & 0.017 \\
$\quad$ Negative & $9(17)$ & $45(83)$ & 0.004 \\
\hline
\end{tabular}

ER: Estrogen Receptor; PR: Progesterone Receptor

Table 2. The correlation of variables with HER2 for the Patients

\begin{tabular}{|c|c|c|c|c|c|c|c|c|c|}
\hline Variable & $\operatorname{Mean}(\mathrm{H}+)$ & $\operatorname{Mean}(\mathrm{H}-)$ & $\mathrm{SD}(\mathrm{H}+)$ & $\mathrm{SD}(\mathrm{H}-)$ & $\mathrm{DF}$ & $\operatorname{SUM}(\mathrm{H}+)$ & $\operatorname{SUM}\left(\mathrm{H}_{-}\right)$ & Test statistics & P-value \\
\hline Age $(\mathrm{T})$ & 44.6 & 48.81 & 7.92 & 8.04 & 128 & & & 1.499 & 0.136 \\
\hline Family History $\left(\mathrm{X}^{2}\right)$ & & & & & 1 & & & 5.519 & 0.036 \\
\hline Tumor Location $\left(\mathrm{X}^{2}\right)$ & & & & & 10 & & & 0.152 & 0.88 \\
\hline Stage(U ) & 99.68 & 56.14 & & & & 4685 & 3830 & 8.224 & 0.012 \\
\hline Lymph node involvement (U) & 90.83 & 51.16 & & & & 4269 & 4246 & 6.063 & 0.024 \\
\hline Number of lymph nodes (U) & 89.9 & 52.16 & & & & 4185.5 & 4329.5 & 6.231 & 0.001 \\
\hline Grade (U) & 99.68 & 46.14 & & & & 4685 & 3830 & 8.22 & 0.021 \\
\hline
\end{tabular}

H: Her2; SD: standard deviation; DF: degree of freedom; T: T test; X²: Chi-square test; U: Mann-Whitney test 
involved lymph nodes in axillaries, grade and hormone receptor status with HER2-neu oncogene status had a direct correlation, but tumor location, age and histological characteristics didn't have significant correlation (Table 2).

We found significant correlation between HER2 with ER and PR and also HER2 with ER,PR negative $(\mathrm{P}<0.05)$, but was no significant correlation between HER2 and ER,PR positive $(\mathrm{P}>0.05)$ (Table 3).

\section{Discussion}

$\mathrm{BC}$ is a malignant tumor that is observed mainly in women. It is noteworthy, prognostic factors in breast cancer are indicators that reflect the individual characteristics of the tumor and the patient (Bailey, 1998). Analysis and evaluation of these factors play a fundamental role in selection of the most effective cancer-specific therapy with the least unnecessary toxic effects produced by inadequate treatment regimens. By enabling prediction of the prognosis, these factors also contribute directly to prolonging survival of women diagnosed with breast cancer, and they have an impact, especially in the short term, on breast cancer mortality (Kurebayashi, 2007). In two studies, HER2-neu amplification was identified in $38 \%$ of 50 patients (Mirtavoos Mahyari et al., 2014) and $23.0 \%$ (Doval et al., 2015). This percent in our patients was $36 \%$. In our study similar to other studies, (Gullick, 1990; Perren, 1991) we found a statistically significant correlation between age and HER2-neu overexpression. Family history of BC significantly associated with elevated HER2 levels in the tumors (Lehrer, 1995) like to our study. Histological grade and nuclear grade are fundamental prognostic factors that over time have been shown to play an important role (White et al., 2003). In a survey of 165 cases of BC, percent of tumor grade for Grade I, Grade II and Grade III was $8.3 \%, 60.8 \%$ and $30.8 \%$, respectively (Seshie et al,. 2015). In this study, Grade I was $13 \%$, Grade II was $77 \%$ and Grade III was $10 \%$. A study claimed that no significant difference regarding HER2-neu status was seen in clinic pathological characteristics of patients (Mirtavoos Mahyari et al., 2014), but in contrast; we found significant relationship between grade and stage with HER2-neu and stage $(\mathrm{P}<0.05)$. It should be noted that lymph node metastases are the most significant prognostic factors in patients with breast carcinoma (Olszewski et al., 2009). A study reported that HER-2/neu protein overexpression is determined by immunohistochemistry correlated with lymph node status and breast cancer tumor grade (Berger et al., 1988). Of $100 \mathrm{BC}$ cases, 89 cases had invasive ductal carcinomas, 4 cases had invasive lobular carcinomas and 7 had other types (Chuangsuwanich et al., 2014). Of 130 cases in our study, 104 had invasive ductal carcinomas, 16 had invasive lobular carcinomas and 10 had other types. The analysis among 1,284 breast cancer patients in India showed that $63.4 \%$ women were ERand/or PR-positive and only $23.1 \%$ women were HER2positive. Overall, $23.7 \%$ women were triple negative and $10.0 \%$ tumors were both ER-/HER2-positive. We found $53.1 \%$ PR-positive and $62.1 \%$ ER-positive in our study. Our results found a significant inverse association between HER2 expression and ER/PR expression ( $p$
$<0.0001$ ) (Doval et al., 2015). However, a recent study by Dawood et al. showed that among metastatic breast cancer patients who were HER2+, more HR-patients benefited from trastuzumab treatment compared to $\mathrm{HR}+$ patients. We found significant relationship between HER2-neu with ER or PR and also with ER-/PR-negative $(\mathrm{P}<0.05)$, but not significant relationship between HER2-neu and ER-/PR-positive $(\mathrm{P}>0.05)$. Two other studies showed $10.0 \%$ tumors were both ER and HER2 positive. ER and PR positivity was significantly associated with HER2negative status $(\mathrm{P}<0.0001)$ (Doval et al., 2015). ER, PR and HER2-neu receptor positivity was $32.1 \%, 25.6 \%$ and $25.5 \%$ respectively (Seshie et al., 2015). Herein; ER, PR and HER2 positivity were $51 \%, 65 \%$ and $36 \%$, respectively. It is noteworthy in anatomy of breast that tumor location in 82 our patients are located in the upper part of the breast, in upper outer (57 of 82) specially.

In conclusion HER2-neu is independent risk factor that it can be a good prognostic factor and also important predictive factor. We recommend that it be evaluated for all different types of $\mathrm{BC}$.

\section{References}

Bailey JA (1988). Concise dictionary of medical-legal terms. New York, The Parthanon Publishing Group.

Berger MS, Locher GW, Saurer S, et al (1988). Correlation of c-erb B2 gene amplification and protein expression in human breast carcinoma with nodal status and nuclear grading. Cancer Res, 48, 1238-43.

Chuangsuwanich T, Pongpruttipan T, O-Charoenrat P, et al (2014). Clinicopathologic features of breast carcinomas classified by biomarkers and correlation with microvessel density and VEGF expression: a study from Thailand. Asian Pac J Cancer Prev, 15, 1187-92.

Dawood S, Broglio K, Buzdar AU, et al (2010). Prognosis of women with metastatic breast cancer by HER2 status and trastuzumab treatment: an institutional-based review. J Clin Oncol, 28, 92-8.

Dorr FA (1993). Prognostic Factors Observed in Current Clinical Trials. Cancer, 71, 2163-8.

DovalDC, Sharma A, Sinha R, et al (2015). Immunohistochemical profile of breast cancer patients at a tertiary care hospital in New Delhi, India. Asian Pac J Cancer Prev, 16, 4959-64.

Dowsett M, Dunbier A (2008). Emerging biomarkers and new understanding of traditional markers in personalized therapy for breast cancer. Clin Cancer Res, 14, 8019-26.

Gullick WJ (1990). New developments in the molecular biology of breast cancer. Eur J Cancer, 26, 509-510.

Hartmann LC, Ingle JN, Wold LE, et al (1994). Prognostic value of c-erbB2 overexpression in axillary lymph node positive breast cancer. Results from a randomized adjuvant treatment protocol. Cancer, 74, 2956-63.

Hu P, Margolis B, Skolnik EY, et al (1992). Interaction of phosphatidylinositol 3-kinase-associated p 85 with epidermal growth factor and platelet-derived growth factor receptors. Mol Cell Biol, 12, 981-90.

Ji H, Lipponen P, Aaltomaa S, et al (1993). c-erbB-2 oncogene related to 533 expression, cell proliferation and prognosis in breast cancer. Anticancer Res, 13, 1147-52.

Klijin JGM (1991). Clinical parameters and symptoms for the progression to endocrine independence of breast cancer. In: mechanisms of progression to hormone-independent growth of breast and prostatic cancer. Carnfoth: The Parthenon Publishing Group, 11-9. 
Kurebayashi J (2007). Current clinical trials of endocrine therapy for breast cancer. Breast Cancer, 14, 200-214.

Lehrer S, Lee P, Tartter P, Shank B, et al (1995). Breast cancer and family history: a multivariate analysis of levels of tumor HER2 protein and familyhistory of cancer in women who have breast cancer. Mt Sinai J Med, 62, 415-8.

Mirtavoos Mahyari H, Khosravi A, Mirtavoos Mahyari Z, et al (2014). Overexpression of HER2/neu as a prognostic value in iranian women with early stage breast cancer; a single institute Study. Iran Red Crescent Med J, 16, 16005.

Mohammadi S, Sulaiman S, Koon PB, et al (2013). Association of nutritional status with quality of life in breast cancer survivors. Asian Pac J Cancer Prev, 14, 7749-55.

Qin T, Yuan ZY, Peng RJ, et al (2015). Clinicopathologic characteristics and prognostic factors for HER2 positive patients with metastaticbreast cancer in southern China. Arch Med Sci, 11, 544-50.

Payandeh M,Sadeghi M, SadeghiE, et al (2015). Clinicopathology figures and long-term effects of tamoxifen plus radiation on survival of womenwith invasive ductal carcinoma and triple negative breast cancer. Asian Pac J Cancer Prev, 16, 4863-7.

Perou C, Sorlie T, Eisen M, et al (2000). Molecular portraits of human breast tumours. Nature, 406,747-52.

Perren TJ(1991). c-erbB-2 oncogene as a prognostic marker in breast cancer. Br J Cancer, 63, 328-32.

Olszewski WP, Szumera-Cieckiewicz A, Piechocki J, et al (2009). The characteristics of the sentinel lymph node metastasis in predicting the axillary lymph node status in patients with breast carcinoma. Pol J Pathol, 60, 138-43.

Seshie B, Adu-Aryee NA, Dedey F, et al (2015). A retrospective analysis of breast cancer subtype based on ER/PR and HER2 status in Ghanaian patients at the Korle Bu Teaching Hospital, Ghana. BMC Clin Pathol, 15, 14.

Sotiriou C, Neo S, McShane L, et al (2003). Breast cancer classification and prognosis based on gene expression profiles from a population-based study. Proc Natl Acad Sci USA, 100, 10393-8.

Yan J, Liu XL, Han LZ, et al (2015). Relation between Ki-67, ER, PR, Her2/neu, p21, EGFR, and TOP II- $\alpha$ expression in invasive ductal breast cancer patients and correlations with prognosis. Asian Pac J Cancer Prev, 16, 823-9.

White J, Morrow M, Moughan J, et al (2003). Compliance with breast conservation standards for patients with early stage breast carcinoma. Cancer, 97, 893-904. 